\title{
ESTUDO DE CASO DE CICLONE EXTRATROPICAL SOBRE A AMÉRICA DO SUL: SENSIBILIDADE DAS ANÁLISES
}

\author{
DAVID MENDES ${ }^{1}$, RILDO GONÇALVES DE MOURA ${ }^{2}$, \\ MONICA CRISTINA DAMIÃO MENDES ${ }^{2}$
}
${ }^{1}$ Centro de Ciência do Sistema Terrestre/Instituto Nacional de Pesquisas Espaciais (CCST/INPE)
${ }^{2}$ Centro de Previsão de Tempo e Estudos Climáticos/Instituto Nacional de Pesquisas Espaciais
(CPTEC INPE), Cachoeira Paulista - SP, Brasil
david.mendes@cptec.inpe.br, rildo.moura@cptec.inpe.br , monica.damiao@cptec.inpe.br

Recebido Novembro 2007 - Aceito Agosto 2009

\begin{abstract}
RESUMO
A trajetória e a energética de um ciclone extratropical são analisadas usando as reanálises do NCEP/ NCAR, em comparação com as saídas do modelo CPTEC/COLA (T126L28) com análises do GPSAS. A analise da energética e da trajetória de um ciclone, formado no leste da Argentina em 23 de Agosto de 2005, mostrou diferenças significativas entre as reanálises e o modelo, principalmente na sua trajetória e magnitude.

A comparação da evolução do ciclone extratropical entre as reanálises e o modelo, mostrou alguns resultados consideráveis, tais como: maior diferença na intensidade da pressão central do ciclone extratropical; maiores diferenças na Energia Cinética após a máxima intensidade do ciclone, e diferenças marcantes no posicionamento do ciclone extratropical.
\end{abstract}

Palavra-chave: reanálises, análises, ciclone extratropical, modelagem.

\begin{abstract}
CASE STUDY OF EXTRATROPICAL CYCLONE OVER THE SOUTH AMERICA: SENSIBILITY OF ANALYSIS

The trajectory and the energetic of extratropical cyclones are analyzed using the NCEP/NCAR reanalysis in comparison with the model outputs CPTEC/COLA(T126L28) with GPSAS analysis. The analysis of the energetic and path of the cyclone formed over East Argentina on 23 August 2005 showed significant differences between the reanalysis and the model, especially in its track and magnitude. The comparison of the extratropical cyclone evolution, between reanalysis and the model, showed some considerable results such as: greater difference in the central pressure intensity of the extratropical cyclones; larger differences in kinetic energy after maximum cyclone intensity and a striking difference in the extratropical cyclone position.
\end{abstract}

Keywords: reanalysis, analysis, extratropical cyclone, modeling.

\section{INTRODUÇÃO}

Os ciclones extratropicais são sistemas de baixa pressão caracterizados por grandes regiões de circulação ciclônica, cujo período de formação é designado por ciclogênese. O primeiro modelo conceitual sobre ciclones foi apresentado em 1922 por Bjerknes e Solberg (1922), que através de observações à superfície verificaram a presença de sistemas de baixa pressão (depressões), sob a forma de perturbações ondulatórias que se propagam ao longo de um sistema frontal. A partir desde estudo, pesquisadores utilizaram métodos variados para a identificação dessas depressões. Sutcliffe em 1947, através da análise diferencial da divergência do vento horizontal em dois níveis distintos da atmosfera, conseguiu identificar na atmosfera os padrões associados às depressões. Petterssen (1956), dando continuidade às pesquisas de Sutcliffe (1947) e reforçando o 
papel da advecção de vorticidade ciclônica na formação das depressões, ressaltou a importância da região baroclínica à superfície no desenvolvimento de novos sistemas ciclogenéticos.

Mais recentemente, devido o avanço da tecnologia e utilizando métodos automáticos de detecção dos ciclones, Murray e Simmonds (1991), Sinclair (1994), Blender et al., (1997) e Trigo et al., (1999) criaram climatologias mais amplas dos ciclones extratropicais tanto para o Hemisfério Sul (HS) com para o Hemisfério Norte (HN). Esses autores utilizaram em seus estudos algoritmos, que tendem a procurar e encontrar o mínimo e/ou o máximo da Pressão ao Nível Médio do Mar (PNMM) ou a altura geopotêncial à superfície (e.g. Trigo et al., 1999) como forma de detecção de ciclones extratropicais. É importante assinalar, que estas climatologias de ciclones extratropicais criadas a partir de deteç̧ão automática, foram obtidas através das reanálises do National Center for Environmental Prediction/National Center for Atmospheric Research (NCEP/NCAR) e das reanálises do European Centre for Medium Range Weather Forecast (ECMWF), em diferentes resoluções espaciais e temporais.

Outros pesquisadores, enfocando apenas a América do Sul (AS), observaram que esta região é propícia para a formação de ciclones extratropicais (e.g. Taljaard, 1967; Necco, 1982; Satyamurty et al., 1990; Gan e Rao, 1991; Sinclair, 1994; e mais recentemente, Mendes et al., 2007), devido ao fato desta área ser uma região altamente baroclínica e possuir uma fonte de energia e entropia favorável a formação deste sistema.

Taljaard (1967) observou a existência de uma região de máxima ciclogênese localizada sobre o Paraguai e um mínimo de ciclogênese a leste da AS, entre 40 e 50S. Necco (1982) realizou o primeiro estudo sobre as distribuições de ciclones extratropicais na AS, ele identificou a região centrosul da AS, como sendo aquela de maior freqüência de ciclones extratropicais. Posteriormente, Satyamurty et al. (1990), com base em 7 anos de dados de satélite sobre a AS, identificaram a região nordeste da Argentina, Uruguai e sul do Brasil, como aquelas de maior atividade de ciclones extratropicais. Gan e Rao (1991) a partir de cartas de superfície e analisando um período de dados mais longos, 10 anos, verificaram que o inverno (JJA) é a estação do ano de maior atividade ciclônica sobre a AS, contrastando com o resultado obtido por Satyamurty et al. (1990), que assinalaram ser o verão a estação do ano de maior atividade ciclônica.

Sinclair (1994), através das análises do ECMWF para o período de 1980 a 1986, obteve uma climatologia extensa de ciclones extratropicais para o HS (sete anos), tendo identificado dois centros de máxima atividade ciclônica sobre a AS; o primeiro sobre o Uruguai e o segundo sobre a costa sudeste da Argentina, resultados esses, também encontrados por Gan e Rao (1991).
De acordo com Sinclair (1994), os ciclones extratropicais formados sobre o Uruguai ocorrem preferencialmente durante o inverno (JJA), enquanto que no verão (DJF) a região de máxima ocorrência se localiza ao sul da região encontrada para os meses de inverno. Simmonds e Keay (2000) obtiveram a partir de dados de reanálises do NCEP/NCAR, para o período de 1958 a 1997, uma nova climatologia de ciclones extratropicais para o HS, cujos resultados encontrados para a AS confirmaram aqueles obtidos anteriormente por Gan e Rao (1991) e Sinclair (1994).

Dando continuidade aos estudos de ciclones extratropicais sobre a AS e utilizando dados de modelo para condições de contornos distintas, este artigo tem como finalidade verificar a destreza do modelo de circulação global, utilizado no Centro de Previsão de Tempo e Estudos Climáticos (CPTEC), em captar a presença de ciclones extratropicais ocorridos no inverno de 2005 sobre a América do Sul.

\section{DADOS, METODOLOGIA E MODELO}

\subsection{Dados}

Neste trabalho foram utilizados dados para o período de 23 a 26 de agosto de 2005 da pressão ao nível médio do mar (PNMM), componente zonal (u) e meridional (v) do vento e altura geopotencial (Z) em $1000 \mathrm{hPa}$, extraídos das reanálises do NCEP/NCAR (na grade de 2.5x 2.5 lat/lon) e da previsão gerada pelo modelo CPTEC/COLA (na grade de $1.875 \times 1.875$ lat/lon) utilizando as análises do GPSAS (mais detalhe abaixo). A área de estudo neste trabalho compreende a faixa longitudinal/ latitudinal de $60 \mathrm{~S} / 15 \mathrm{~S}$ a $120 \mathrm{~W} / 0$.

\subsubsection{Reanálises NCEP/NCAR}

As reanálises do NCEP/NCAR envolvem a recuperação de informações coletadas sobre a superfície e sobre oceanos através de navios, aviões, radiossondas e Satélites. Essas informações foram e são computadas por meio de um sistema de assimilação de dados que é mantido constantemente atualizado. Esse procedimento elimina possíveis erros associados com a mudança no sistema de assimilação (Kalnay et al., 1996).

\subsubsection{Modelo CPTEC/COLA}

O modelo CPTEC/COLAdescende do modelo de previsão de médio prazo do NCEP, então National Meteorological Center (NMC), denominado pelo NCEP como MRF ("Médium Range Forecasting Model"), cujas compilações espectrais (parametrizações dos processos físicos) foram desenvolvidas 
no NCEP/NCAR, mais precisamente no Geophysical Fluid Dynamics Laboratory (GFDL). Posteriormente, foi feita transferência deste modelo para o Center for Ocean, Land and Atmosphere Studies (COLA), onde foram adicionados cálculos diagnósticos para um melhor entendimento dos processos físicos. Desde a implementação do MRF no NCEP, o modelo sofreu varias mudanças, que vão desde as parametrizações de processos termodinâmicos, às condições de fronteira e contorno distintas. No caso do CPTEC, a versão adotada é a 1.7 do COLA, que tem truncamento romboidal (truncamento triangular na onda zonal de número 62). Ele é uma versão T062L28, isto é, tem uma resolução horizontal de 62 km e 28 níveis na vertical.

\subsubsection{Analises GPSAS}

A análise do GPSAS (Global Physical-space Statistical Analysis System) é um sistema que integra uma previsão do modelo global de 6 horas (primeiro-input) com dados observacionais, para produzir análises e previsões em um processo cíclico, em que a cada novo ciclo, uma nova análise é produzida, de modo que os erros de estimativa dos first-guess e dos dados observacionais sejam minimizados (Guo et. al., 1998).

\subsection{Metodologia}

A metodologia utilizada para a detecção dos ciclones extratropicais, ocorridos entre as 0600 UTC do dia 23 de agosto de 2005 até as 1800 UTC do dia 26 de agosto de 2005, é semelhante àquela proposta por Mendes et. al., (2007), cujo algoritmo de detecção identifica potenciais candidatos a ciclones a partir da verificação de isóbaras fechadas em uma área de $3 \times 3$ ponto de grade. Esta metodologia foi aplicada tanto para os dados extraídos das reanálises como para os dados extraídos do modelo.

\subsubsection{Identificação dos Ciclones}

Para ser considerado um ciclone extratropical é necessário que o mínimo de pressão à superfície satisfaça os seguintes critérios:

1) Ter um valor máximo de 1015 hPa para a pressão central do sistema de baixa pressão;

2) Ter um gradiente médio da pressão de $0.55 \mathrm{hPa} / 250$ $\mathrm{km}$ estimado em uma área de 5 de latitude por 7 de longitude.

De modo a identificar as trajetórias, para cada mínimo de pressão detectado, procura-se o centro mais próximo nos
18 UTC

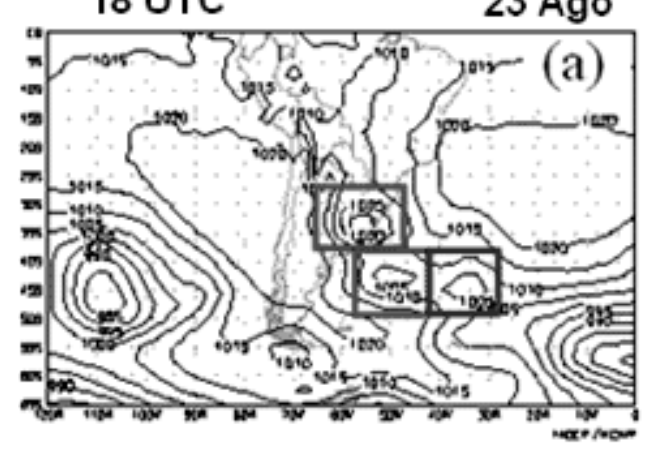

18 UTC

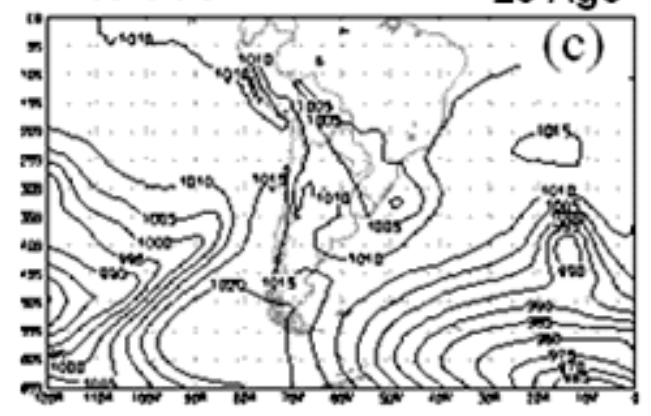

12 UTC

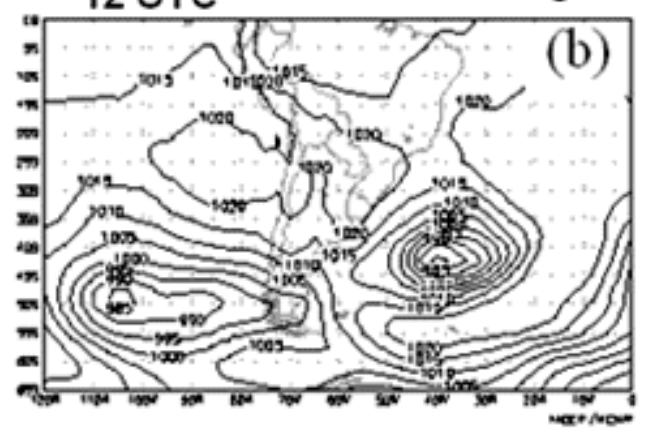

12 UTC

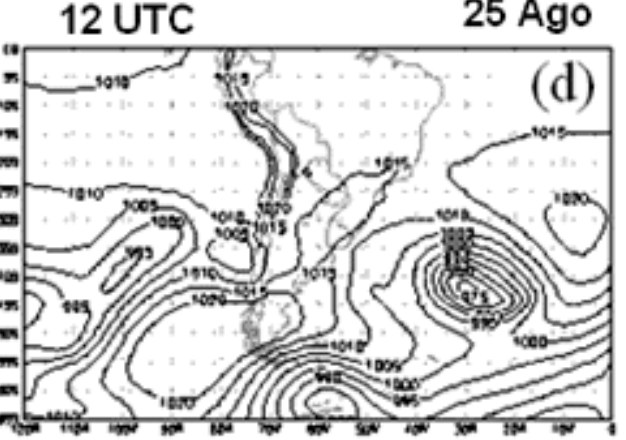

Figura 1 - Evolução espacial da pressão ao nível do mar para o NCEP (a, b), e modelo com GPSAS (c, d), para as 1800 UTC do dia 23 de agosto de 2005 e as 1200 UTC do dia 25 de agosto. 
tempos seguintes, admitindo que a velocidade máxima de cada ciclone extratropical não exceda $33 \mathrm{~km} / \mathrm{h}$ para oeste e $90 \mathrm{~km} / \mathrm{h}$ para qualquer outra direção. Mais detalhes sobre o método de detecção pode ser encontrado em Mendes et al., (2007).

\subsubsection{Cálculo da Energia Cinética}

O estudo da energia cinética por unidade de massa $(k)$, ao longo de uma determinada latitude, é obtido segundo Haltiner e Williams (1980), ou seja:

$$
k=(1 / 2 \pi) \int_{0}^{2 \pi}\left[1 / 2\left(u^{2}+v^{2}\right)\right] d \lambda
$$

sendo que o primeiro termo do segundo membro da equação corresponde a energia cinética do escoamento zonal $\left(u^{2} / 2\right)$, e o segundo termo a energia cinética do escoamento meridional $\left(v^{2} / 2\right)$.

\section{RESULTADOS}

\subsection{Características dos Ciclones}

A Figura 1 mostra a PNMM (a) para o dia 23 de agosto de 2005 às 1800 UTC (ciclogênese), e (c) do dia 25 de agosto de 2005 às 1200 UTC $(\mathrm{b}, \mathrm{d})$ máxima intensidade do ciclone extratropical para a reanálise $(\mathrm{a}, \mathrm{b})$, e para os resultados do modelo (c, d).

No campo da PNMM para a reanálise (Figura 1a), verifica-se a presença de três centros de baixa pressão, o primeiro localizado sobre o Uruguai e os outros dois situados sobre o litoral da AS. Esse resultado diferencia daquele encontrado pelo modelo (Figura 1c), onde às 1800 UTC do dia 23 de agosto, verifica-se apenas uma pequena baixa localizada sobre o litoral sul do Brasil. Na Figura 1b e 1d, os resultados encontrados são semelhantes para as duas séries de dados (reanálise e modelo). A pressão central durante o período de máxima intensidade (Figura 1b e 1d), apresenta diferenças entre a reanálise e o modelo, isto é, no modelo (Figura 1d) a pressão central é mais cavada $(\sim 975 \mathrm{hPa})$, enquanto que para a reanálise (Figura $1 \mathrm{~b}$ ) a pressão central não ultrapassa os $985 \mathrm{hPa}$.

$\mathrm{Na}$ Figura 2 são apresentadas as trajetórias do evento (ciclone extratropical), no período de estudo (23 a 26 de agosto de 2005), para as duas séries de dados (reanálise e modelo), os resultados mostram-se aproximados não só com relação à direção, como também, no posicionamento (latitudelongitude), sugerindo assim uma convergência nos resultados encontrados.

As saídas do modelo (linha em cinza), após 24 horas, apresentam uma diminuição acentuada da pressão até atingir o mínimo de pressão após 42 horas, enquanto que para a reanálise (linha em preto), o mínimo de pressão é tardio em relação ao modelo, isto é, ocorre apenas 66 horas após a formação, apresentando uma taxa de crescimento (aumento da pressão) após o mínimo de pressão (Figura 3).

\subsection{Energia Cinética e Evolução do Evento}

A Figura 4 mostra o campo da energia cinética $(k$, em cores), altura (mgp, linhas vermelhas) e do vetor vento $\left(\mathrm{m} \mathrm{s}^{-1}\right.$, setas) em $1000 \mathrm{hPa}$ para 0000 e 1200 UTC do dia 24 de agosto.

\section{Tracks}

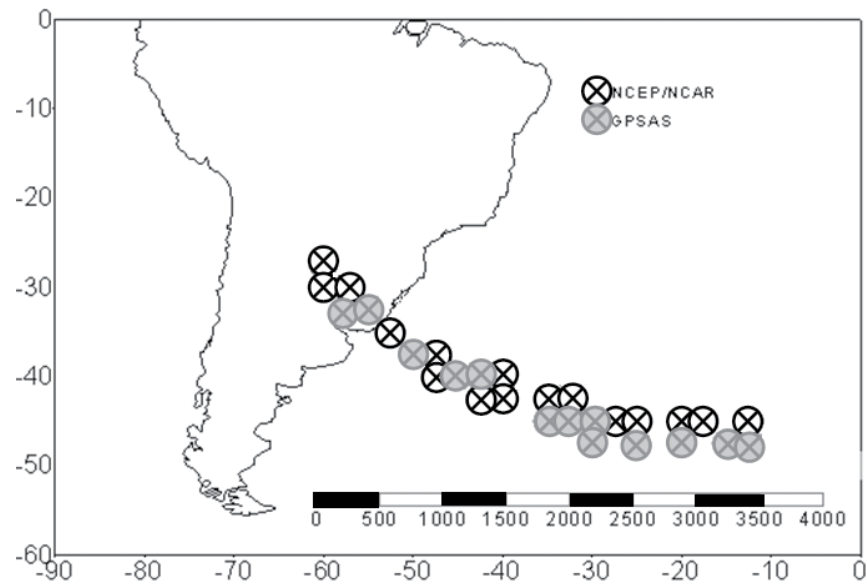

Figura 2 - Trajetória do ciclone extratropical durante o período de estudo. Reanálises (símbolo em preto) GPSAS (simbolo em cinza). A escala representa a distância em $\mathrm{km}$. 
Nota-se para este período que o ciclone extratropical entra em intensa formação para ambas as séries de dados. Na reanálise (Figura 4a) às 0000 UTC do dia 24 de agosto, é possível observar um sistema de baixa pressão bem configurado sobre o litoral do Uruguai, com um máximo de energia cinética $(k)$ localizada no flanco equatorial do ciclone extratropical. Para o modelo (Figura 4b), a circulação de baixa pressão não se apresenta bem configurada, em comparação com aquela vista para a reanálise, mostrando apenas uma intensa região de $k$ sobre o Sul do Brasil. Para as 1200 UTC (Figura 4c, d), verifica-se que há uma uniformidade entre a reanálise e o modelo, cujo sistema de baixa pressão mostra-se bem configurado, apresentado um máximo de $k$ no flanco equatorial da região de baixa pressão.

No dia 25 de agosto de 2005 as 0600 e 1800 UTC (Figura 5a-d), verifica-se uma similaridade entre o modelo e a reanálise, havendo diferença apenas na localização do núcleo do ciclone

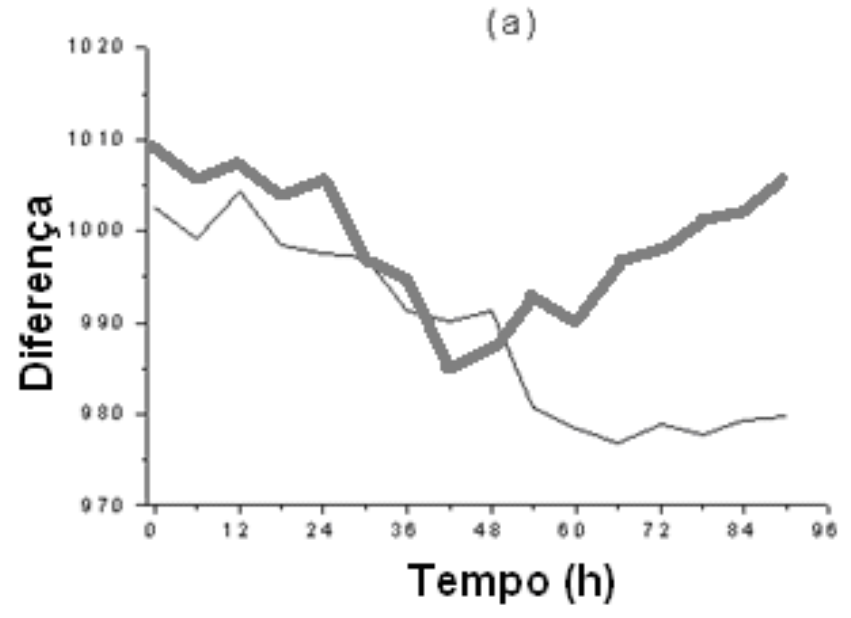

Figura 3 - Evolução temporal da pressão mínima no centro do ciclone extratropical. NCEP (linha em preta) e modelo com GPSAS (linha em cinza).
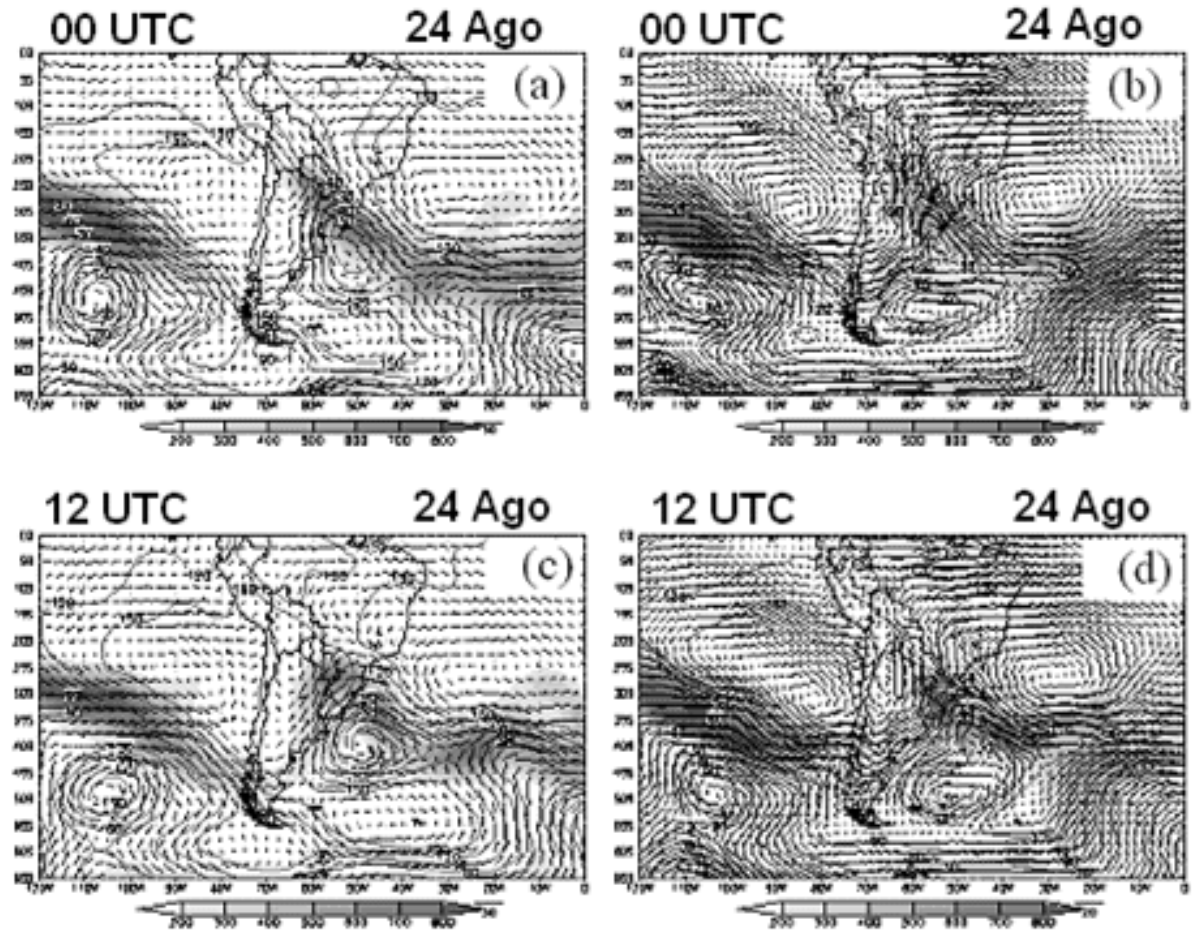

Figura 4 - Evolução espacial da energia cinética $\left(\mathrm{k}, \mathrm{m}^{2} \mathrm{~s}^{-2}\right)$ altura geopotencial $(\mathrm{hPa})$ em $1000 \mathrm{hPa}$ e vetor vento em $1000 \mathrm{hPa}\left(\mathrm{m} \mathrm{s}^{-1}\right)$, para o período de 0000 UTC e 1200 UTC do dia 24 de agosto, $\operatorname{NCEP}(a, b)$ e modelo com GSPAS (c, d). Em cores a energia cinética, em linhas vermelhas a altura geopotencial e em setas o vetor vento. 
extratropical. Vale salientar, que o processo de transferência de energia corrente abaixo, ou seja, "downstream", ocorre principalmente nos altos níveis da atmosfera, onde o vento ageostrófico é praticamente zonal e $k$ apresenta sua maior magnitude (Blackburn, 1985).

Por sua vez, a evolução da energia cinética (Figura 6) apresenta certas similaridades nas primeiras horas (12 horas) para o modelo e a reanálise, no qual a intensidade chega à ordem de $350 \mathrm{~m}^{2} \mathrm{~s}^{-2}$. Na reanálise (linha em preta) após as primeiras 24 horas o ciclone perde intensidade, voltando a máxima atividade 48 horas após a formação $\left(800 \mathrm{~m}^{2} \mathrm{~s}^{-2}\right)$. Esse resultado também é observado no modelo (linha em cinza), porém no caso do modelo, o ciclone não perde intensidade, tendo uma tendência gradual de intensificação.

\subsection{Evolução do Sistema}

Como mencionado na metodologia, a detecção e acompanhamento dos ciclones extratropicais foi criado a partir do método de detecção automático proposto por Mendes et. al. (2007), nesse método, a evolução do sistema é observada sob forma de deslocamento, tempo de vida (horas), velocidade de deslocamento a cada 6 horas $\left(\mathrm{km} \mathrm{h}^{-1}\right)$, máximo decaimento ( $\mathrm{dp} \mathrm{dt}^{-1}$ 6-horas), pressão mínima (hPa), entre outros. Porém, neste trabalho dá-se o enfoque apenas ao tempo de vida (horas), velocidade de deslocamento e distância total percorrida pelo evento.

\section{a.Tempo de vida}

O tempo de vida encontrado para a reanálise tem uma diferença bastante significativa em relação ao verificado no modelo, isto é, o sistema tem um tempo de vida em torno de 70 horas para a reanálise e de 60 para o modelo. Esses resultados aproximam-se com os casos selecionados por Mendes (2006), no inverno, para o HS e AS, cujos tempos de vida são cerca de 74 horas para o HS e 73 horas para o AS.

\section{b. Velocidade de deslocamento}

A Tabela 1 mostra ainda a velocidade média de deslocamento do ciclone extratropical a cada 12 horas. Nota-se que não há uma grande variação na velocidade entre as duas séries de dados (reanálise e modelo). A velocidade média máxima $\left(21 \mathrm{~km} \mathrm{~h}^{-1}\right)$ é verificada entre as 24 e 36 horas após a formação, esse resultado é coerente com aquele encontrado por Mendes
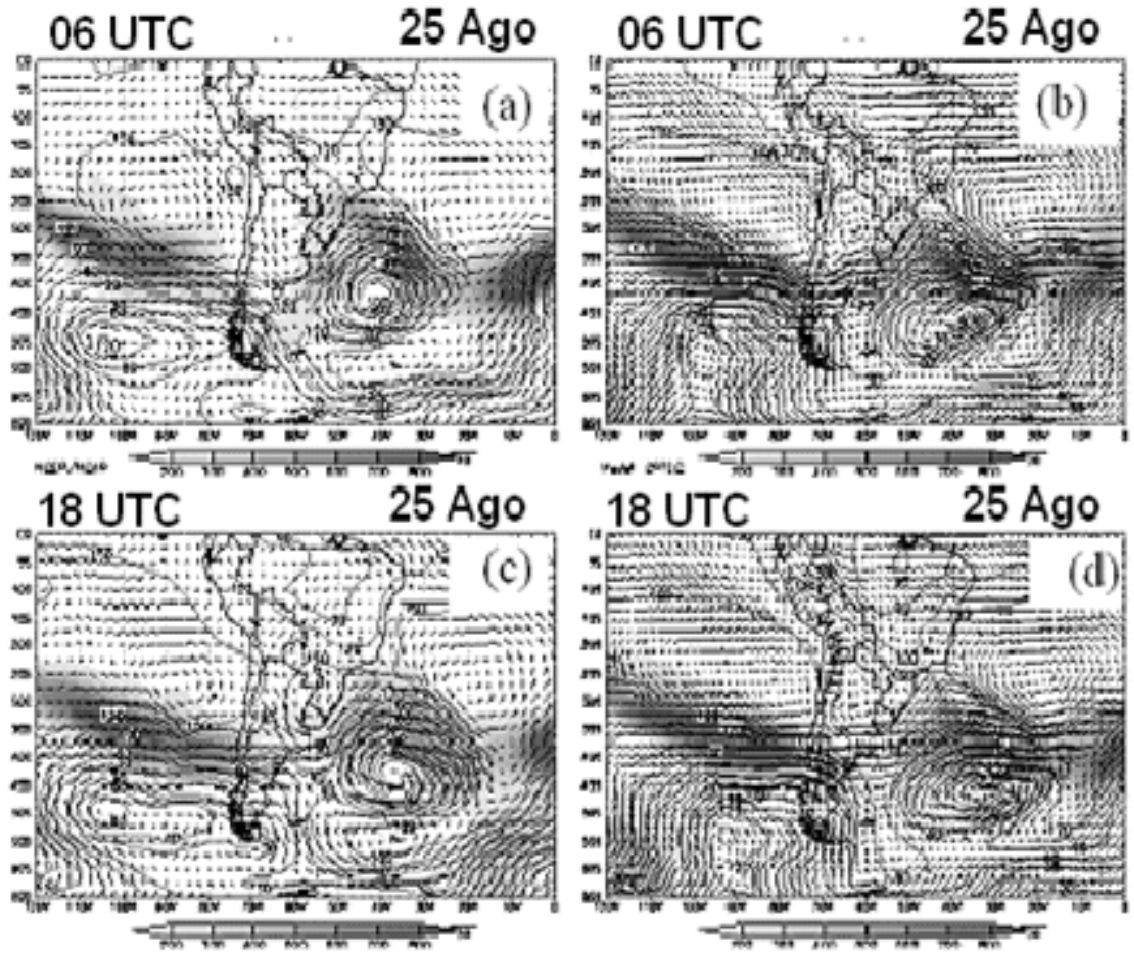

Figura 5 - Assim como para a Figura 4, mas para o período de 0600 UTC e 1800 UTC do dia 25 de agosto de 2005. 


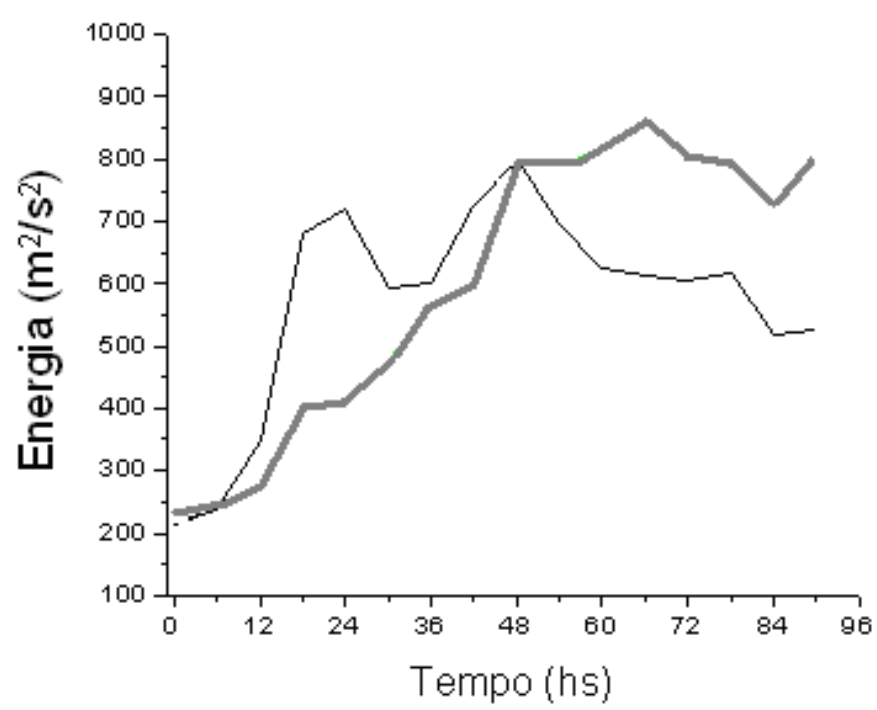

Figura 6 - Assim como para a Figura 3, mas para o caso Energia cinética.

Tabela 1 - Velocidade $(\mathrm{km} / \mathrm{h})$ média do ciclone extratropical durante o período de estudo (12h a 72 horas).

\begin{tabular}{|l|l|l|l|l|l|l|l|}
\hline \multicolumn{7}{|c|}{ Horas (h) } \\
\hline & 12 & 24 & 36 & 48 & 60 & 72 & Média \\
\hline NCEP & 22.2 & 22.1 & 21.5 & 20.1 & 20.4 & 19.7 & 21.0 \\
\hline GPSAS & 22.1 & 22.5 & 22.0 & 21.0 & 20.1 & 19.4 & 21.2 \\
\hline
\end{tabular}

(2006). Mendes (2006) verificou através de uma climatologia para 55 anos de dados da reanálise do NCEP/NCAR, que os ciclones extratropicais de inverno na AS apresentam uma velocidade média de aproximadamente $21 \mathrm{~km} \mathrm{~h}^{-1}$, média muito parecida com a observada no estudo aqui realizado.

\section{c.Distância total percorrida pelo ciclone extratropical}

A distância total, percorrida pelo ciclone extratropical (Tabela 1), apresenta diferenças entre reanálise e o modelo. $\mathrm{Na}$ reanálise a distância média percorrida é de aproximadamente $3551 \mathrm{~km}$ (Figura 2), enquanto que para o modelo a distância é de $3580 \mathrm{~km}$. Os resultados aqui encontrados discordam dos resultados obtidos por Mendes (2006), ou seja, a distância total é superior à média encontrada por Mendes (2006). Ele verificou que raramente ciclones extratropicais formados sobre a AS percorrem mais de $2500 \mathrm{~km}$.

\section{CONCLUSÃO}

Os resultados encontrados mostram que os ciclones extratropicais originados sobre a AS, apresentam algumas características idênticas as observadas em outras regiões do HS (e.g. Sinclair,
1995; Simmonds e Keay, 2000), cuja região preferencial de ciclogênese ocorre a leste da Argentina, Uruguai e Sul do Brasil, sendo o inverno a estação do ano com maior atividade e o verão com menor atividade de ciclones.

Comparando a evolução do ciclone extratropical entre a reanálise do NCEP/NCAR e o modelo CPTEC/COLA gerados a partir das análises GPSAS, têm-se as seguintes conclusões:

1)Não há grandes diferenças no posicionamento da trajetória do ciclone extratropical (Figura 2);

2)A maior diferença na intensidade da pressão central entre o modelo e as reanálises ocorre após a máxima intensidade, $\sim 42$ horas depois da formação (Figura 3);

3)A maior diferença na Energia Cinética $(k)$ ocorre também após a máxima intensidade, 48 horas depois da formação (Figura 6).

Em relação à trajetória (Figura 2) e a velocidade média de deslocamento (Tabela 1), as maiores diferenças entre o modelo e a reanálise ocorrem após 24 horas. Entre as primeiras 12 e 24 horas, as dissimilaridades são pequenas e quase insignificantes. Para a velocidade de deslocamento existe pouca variação na média, $21.0 \mathrm{~km} \mathrm{~h}^{-1}$ para a reanálise e $21.2 \mathrm{~km} \mathrm{~h}^{-1}$ para o modelo.

Finalmente, para uma melhor confirmação da "destreza" do CPTEC/COLA com análise do GPSAS, sugerimos a elaboração de estudos complementares em relação a eventos sinóticos sobre o HS, em especial na AS.

\section{AGRADECIMENTOS}

O autor agradece ao Centro de Previsão de Tempo e Estudos Climáticos (CPTEC) pela disponibilidade das saídas 
(output) do Modelo de Circulação Geral da Atmosfera (MCGA), assim como aos revisores pelas sugestões.

\section{REFERÊNCIAS BIBLIOGRÁFICAS}

BJERKNES, J.; SOLBERG, H. Life Cycle of Cyclones and the Polar Front Theory of Atmospheric Circulation. Geophysical, v. 3, n. 1, 325p., 1922.

BLENDER, R., FRAEDRICH, K., LUNKEIT, F. Identification of cyclone-track regimes in the North Atlantic. Quart. J. Roy. Met. Soc., v. 123, n. p239, 727-741, 1997.

BLACKBURN, M. Interpretation of ageostrophic winds and implications for jet stream

maintenance. J. Atmos. Sci, v. 42, n. 23, p. 2604-2620, 1985.

GAN, M. A., RAO, V. B. Surface cyclogenesis over South America. Mon. Wea. Rev., v. 119, n. 5, p. 1293-1302, 1991.

GUO, J., et al. Documentation of the Physical-space Statistical Analysis System (PSAS) Part II: The factored-operator formulation of error covariances, DAO Office Note, Data Assimilation Office, Goddard Space Flight Center, abr. 1998.

HALTINER, G. J., WILLIAMS R. T. Numerical Prediction and Dynamic Meteorology. 2d ed. John Wiley and Sons, pp. 477, 1980.

KALNAY, E., et al. NCEP/NCAR 40-year reanalysis project, Bull. Am. Meteorol. Soc., v. 77, n. 3, p. 437-471, 1996.

MENDES, D. Regimes de circulação no Atlântico Sul e sua relação com a localização e intensidade de sistemas ativos e com o balanço de vapor na região. Tese de Doutorado, Univ. de Lisboa, 125p, 2006.

MENDES, D., SOUZA, E. P., TRIGO, I. F., MIRANDA, P. M. A. On Precursors of South-American Cyclogenesis. Tellus
A, v. 59, n. 1, p. 114-121, 2007.

MURRAY, R.J., SIMMONDS, I., A numerical scheme for tracking cyclone centres from digital data. Part I: development and operation of the scheme. Aust. Met. Mag., v. 39 , n. 2, p. 155-166, 1991.

NECCO, G. Comportamiento de vortices ciclonicos en el area Sudamericana durante el FGGE : Ciclogenesis. Meteorologica, v.13, n. 1-2, p. 7-19, 1982.

ORLANSKI, I., KATZFEY, J. The life cycle of a cyclone wave in the Southern Hemisphere. Part I: Eddy energy budget. J. Atmos. Sci., v. 48, n. 17, p. 1972-1998, 1991.

PETTERSSEN, S. Weather analysis and forecasting. Vol. I: Motion and motion systems. New York, McGraw Hill Book Co. Inc., 428p, 1956.

SATYAMURTY, P., FERREIRA, C. C., GAN, M. A. Cyclonic vortices over South America. Tellus A, v. 42A, n. 1, p. 194-201, 1990.

SIMMONDS, I., KEAY, K. Mean southern hemisphere extratropical cyclone behavior in the 40-year NCEP-NCAR reanalysis, J. Climate, v. 13, n. 5, p. 873-885, 2000.

SINCLAIR, M.R. An objective cyclone climatology for the Southern Hemisphere. Mon. Wea. Rev. v. 122, n. 10, p. 2239-2256, 1994.

SUTCLIFFE, R. C., A Contribution to the problem of development. Quart. J. Roy. Meteor. Soc., v. 73, n. 5, p. 370-383. 1947.

TALJAARD, J. J., Development, distribution and movement of cyclones and anticyclones in the Southern Hemisphere during the IGY. J. appl. Met., v.6, n. 6, p. 973-987, 1967.

TRIGO, I. F., DAVIES T. D,. BIGG G. R, Objective climatology of cyclones in the Mediterranean region. J. Climate, v12, n. 10, p. 1685-1696, 1999. 